\title{
Optomechanics Outside the Lab: Prototyping and Field-testing a Whispering Gallery Mode Accelerometer
}

\author{
Ying Lia Li and P. F. Barker \\ Department of Physics \& Astronomy, University College London, Gower Place, London, WC1E 6BT, United \\ Kingdom \\ ying.li.11@ucl.ac.uk
}

\begin{abstract}
The dispersive and dissipative optomechanical coupling between a whispering gallery mode (WGM) resonance and the motion of the WGM cavity is used to measure acceleration. We describe the prototype assembly and results from the outdoor field-trials.
\end{abstract}

OCIS codes: $120.0120,140.3948$.

Optomechanical systems show great promise for ultraprecise measurements of acceleration that surpass the performance of commercial systems. We previously demonstrated an optomechanical accelerometer by exploiting optical whispering gallery mode (WGM) resonances within a spherical cavity attached to movable cantilever [1]. The main sensing region consists of a silica microsphere WGM resonator attached to a cantilever, known as the microspherecantilever, that is evanescently coupled to a tapered waveguide. Deflections of the cantilever in response to acceleration alter the relative coupling distance between the sphere and the waveguide $\left(d_{0}\right)$, as shown in Fig. 1 (a), in turn causing a measurable shift of the WGM resonance and a broadening of the resonance linewidth. This dispersive and dissipative optomechanical coupling allows detection of accelerations to the micro- $\mathrm{g} \mathrm{Hz}^{-1 / 2}$ level $\left(\mathrm{g}=9.81 \mathrm{~ms}^{-2}\right)$. Commercializing such a sensor requires feasibility studies outside the laboratory environment. Here we discuss the assembly of a portable prototype powered from batteries and automated with a field programmable gate array (FPGA). The prototype has a noise density of approximately 40 micro- $\mathrm{g} \mathrm{Hz}^{-1 / 2}$, almost ten times larger than the lab system demonstrated previously [1] in order to test its performance and survivability during field-trials rather than obtaining the highest sensitivity.

(a)

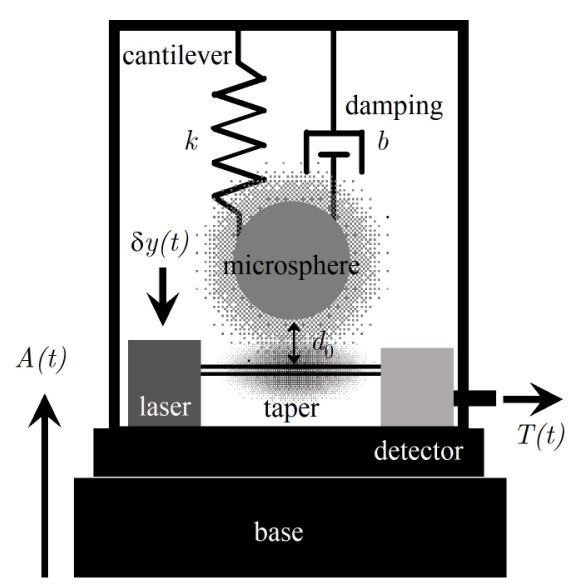

(b)

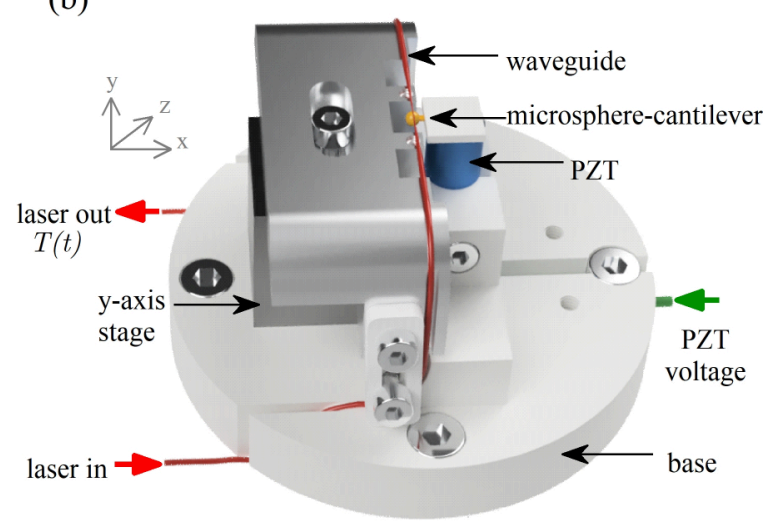

Fig. 1. (a) An acceleration, $A(t)$, in the vertical direction, applied to the base of the setup causes the cantilever to deflect by a distance $\delta y(t)$ away from $d_{0}$. (b) Illustration of the mechanical mounting of the WGM sensor components.

An important feature of the prototype is substantial reduction in external mechanical responses arising from mounts or translation stages. The microsphere-cantilever and the tapered fiber are therefore aligned and mounted using only 
four separate mechanical elements, shown in Fig. 1 (b). The microsphere-cantilever is bonded to a piezostack (PZT) which is screwed into a CNC machined base. A 1-D manual translation stage is also secured onto the base allowing for coarse alignment $(>10 \mu \mathrm{m})$ of the mounted taper fiber with respect to the microsphere-cantilever. The PZT provides fine adjustment of the coupling distance such that $d_{0}<0.6 \mu \mathrm{m}$. When used for applications such as navigation, it is important for accelerometers to have long-term stability reducing the need for re-calibration. Due to the use of an open-loop PZT to translate $d_{0}$ we observe a slow drift due to the poor DC stability and creep of PZTs [2]. This creates a false deflection which can result in substantial DC acceleration errors. Employing a closed-loop feedback scheme is therefore crucial to ensure $d_{0}$ is kept stable. To this end, we send the WGM sensor output through a digital $100 \mathrm{~Hz}$ low pass filter which is then processed by a digital PID loop with only proportional feedback enabled. This feedback signal is sent to the PZT supporting the microsphere-cantilever in order to stabilize $d_{0}$ and is controlled by the FPGA.

The prototype accelerometer is mounted onto a 4-wheel drive truck driven at average speeds of $20 \mathrm{~m} \mathrm{hr}^{-1}$ over curbs, railway sleepers and ditches to produce large accelerations and shocks in the vertical (y-axis), coinciding with the sensing axis of the accelerometer. The raw data from one run is shown in Fig. 2 with the inclusion of a commercial IEPE accelerometer output for comparison. We note that the IEPE sensor requires recovery after shocks exceeding $\pm 50 \mathrm{~g}$ which is a combination of the RC time constant of the sensor and signal conditioner. The WGM recovers almost immediately, with small DC offsets due to the fixed gain of the closed-loop feedback.

(a)

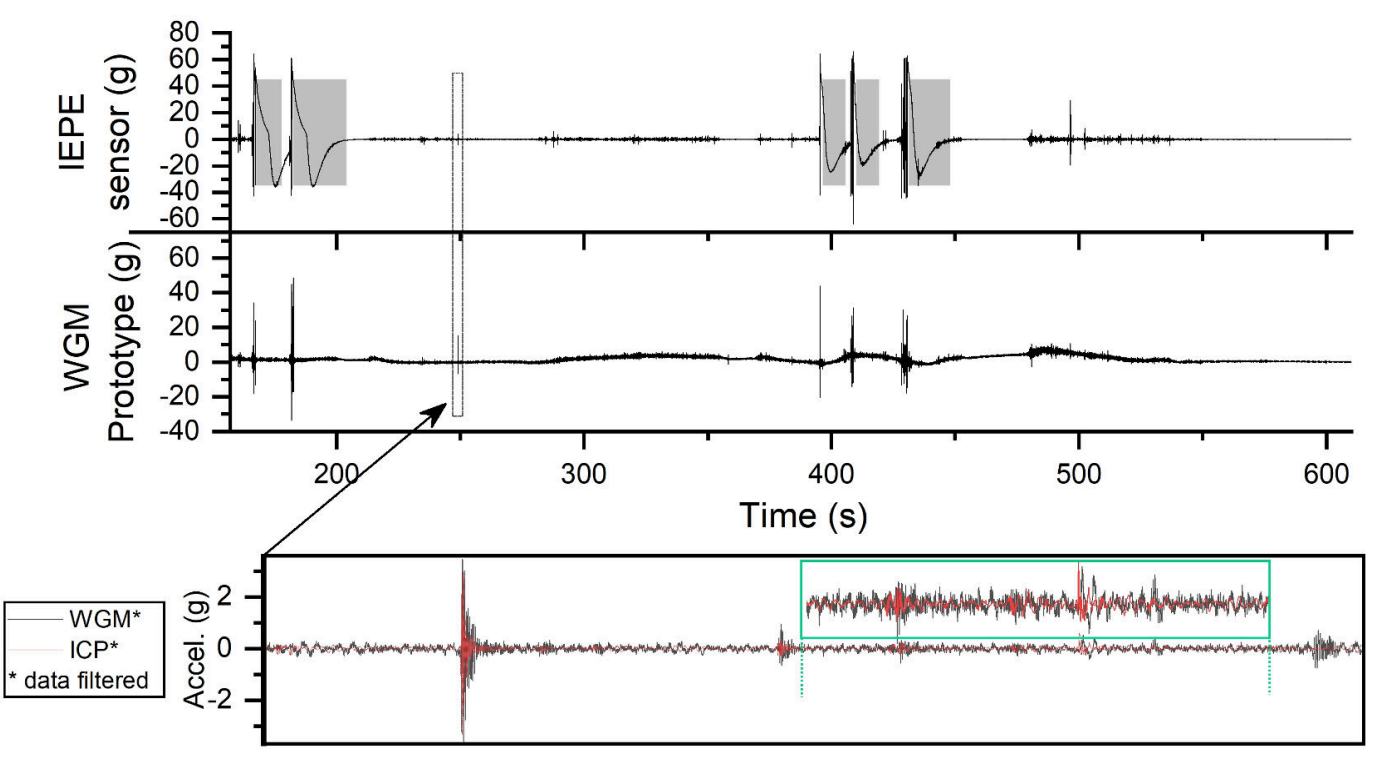

Fig. 2. (a) Prototype data with closed-loop feedback showing a return to $d_{0}$ even after shocks exceeding $\pm 60 \mathrm{~g}$, verified by the IEPE sensor (AC response $<5 \mathrm{~Hz}$ only, with a recovery time shaded in grey). Past $\pm 6 \mathrm{~g}$ the WGM prototype is pushed into an uncalibrated \& non-linear response but recovers on a negligible time-scale. (b) Comparison of a segment of (a) where a band-pass filter $(0.1$ $-5 \mathrm{kHz}$ ) is applied \& data $>|6| \mathrm{g}$ is removed; both sensors agree well to applied shock and engine vibration.

Further analysis of the trial data will be presented as well as improvements for the next prototype iteration and further required subsystem engineering.

\section{References}

1. Y. L. Li and P. F. Barker, "Characterization and Testing of a Micro-g Whispering Gallery Mode Optomechanical Accelerometer," arXiv:1805.07130 [physics.app-ph], May 2018.

2. H. Jung and D-G. Gweon, "Creep characteristics of piezoelectric actuators," Rev. of Sci. Instruments 71(4), 1896-1900 (2000). 